\title{
Detection of gear faults in variable rotating speed using variational mode decomposition (VMD)
}

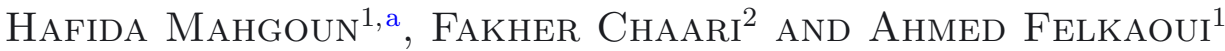 \\ ${ }^{1}$ Laboratoire de Mécanique de Précision Appliquée (LMPA), Institut d'Optique et Mécanique de Précision, Université Ferhat \\ Abbas Sétif 1, Algérie \\ ${ }^{2}$ Laboratoire de Mécanique, Modélisation et Production (LA2MP), Ecole Nationale d’ingénieurs de Sfax, Tunisie
}

Received 14 February 2015, Accepted 6 August 2015

\begin{abstract}
The ensemble empirical mode decomposition (EEMD) was largely used in the diagnosis of the rotating machines, this method could detect the defect at an early stage in the case of non variable speed or slightly variable, but when the speed of the machine varies in acceleration or deceleration the use of the EEMD under these conditions shows a limitation with the detection of the impulses, that are influenced by the presence of the mode mixing, and the end effect. To detect the shocks due to the defect where the variation of speed is forced by the working conditions, we propose to use the Variational Mode Decomposition (VMD) which was recently proposed by Konstantin Dragomiretskiy. This method gave promising results in the detection of the defects on machine elements under non stationary conditions imposed by the variation of speed and torque. In this work, first we show by simulated signal the advantage of VMD compared to the EEMD in the detection of impulses in the case of variable speed and load. Then, we analyze vibration signals given by a dynamic modeling of a gear transmission in the case of non stationary load and speed, for healthy gear and two different of localized faults (early and advanced). The modes are extracted using VMD and followed by calculation of spectrogram and statistics values, which give more information about the defect and allow us to detect it at an early stage.
\end{abstract}

Key words: Vibration analysis / non stationary operation / time-varying frequency / variational mode decomposition (VMD) / ensemble empirical mode decomposition (EEMD) / intrinsic mode functions (IMFs) / gear fault detection / rotating machines

\section{Introduction}

Gears are mechanisms widely used for power transmission in rotating machinery. The malfunctions and defects of gears are inevitable. The faulty gear is usually the major source of noise and vibration [1] and may result in the abnormal operation and failure of the system. The early detection of gear faults is very important to prevent the system from damage. Vibration analysis is the most commonly used method for diagnosing gear faults since the vibration signals give plentiful information related to machines $[1,2]$.

When a local gear fault occurs, both amplitude and phase of the tooth meshing vibration signals are modulated [3-5]. If the rotating speed of the shaft is invariable the gear-fault-induced modulation phenomenon manifests as frequency sidebands equally spaced around the meshing frequency and its harmonics in vibration spectra [3-5]. However, under variable rotating speed of the shaft, the

${ }^{a}$ Corresponding author: mahafida006@yahoo.fr meshing frequency and its harmonic and the sidebands vary with time and hence the vibration signal is nonstationary $[2,6-8]$. The use of methods which decompose the signal into bands is very useful in these situations.

The ensemble empirical mode decomposition (EEMD) method [9] was largely applied in fault diagnosis of rotating machinery $[5,7,8,10,11]$ because it does not use a priori determined basis functions and can iteratively decompose a complex signal into a finite number of intrinsic mode functions (IMFs). Each resulting elementary component IMF can represent the local characteristic of the signal $[9,12]$. But all these papers used the EEMD to analyze signals collected from test bench which works under stationary conditions, where the speed of the shaft is constant or slowly variable.

Recently, Variational Mode Decomposition (VMD) method was proposed as a multiresolution technique [13] that overcomes some limits of the EEMD [14-17] which highly depends on the choice of an interpolation method; the number of iterations. VMD technique faces the difficulty of having no mathematical model besides its 


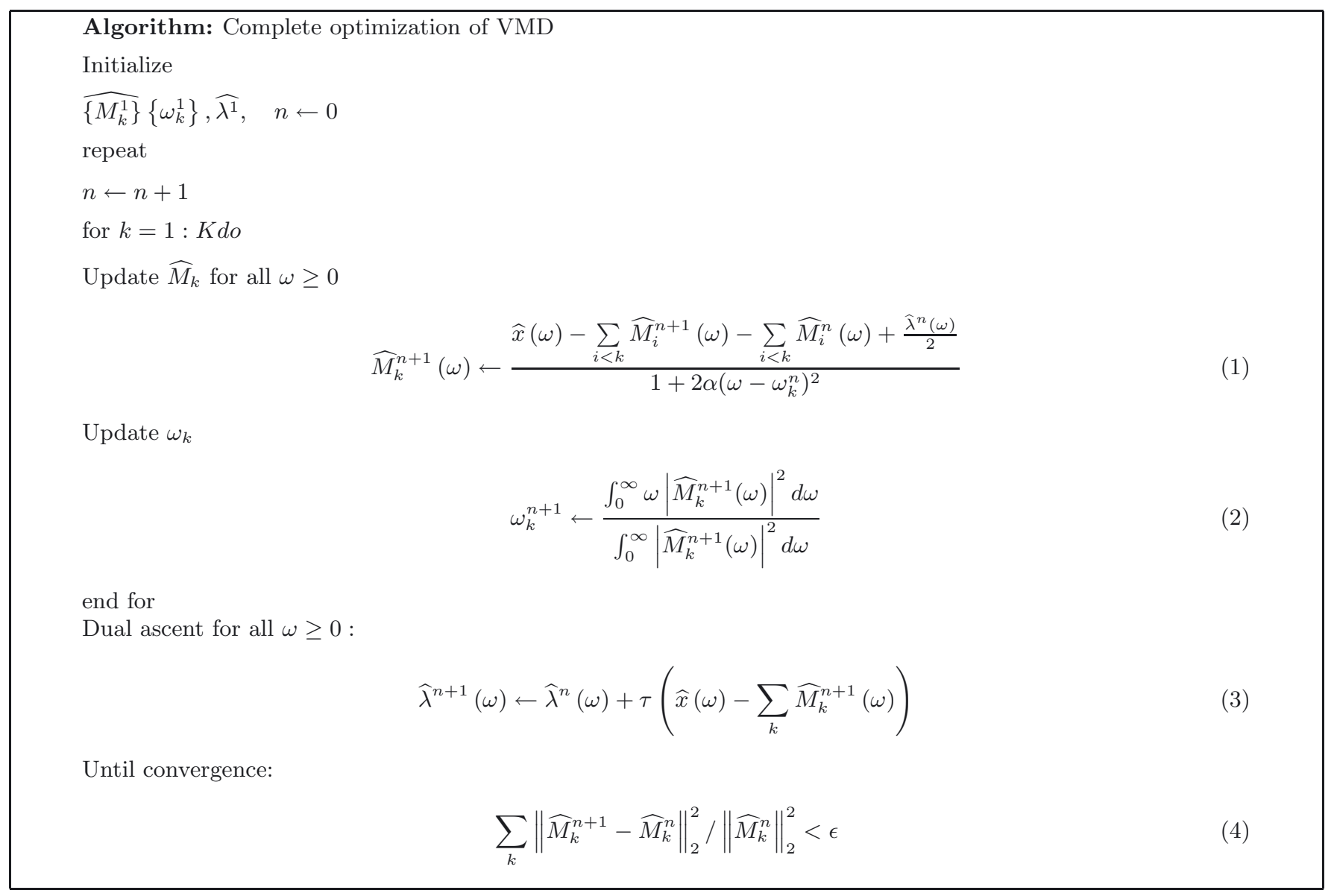

Fig. 1. Algorithm of VMD [13].

algorithm and other problems like sensitivity to noise, the choice of sampling frequency $[17,19]$ and the problem of mode mixing $[14,16,17]$. VMD algorithm is very similar to empirical mode decomposition (EMD) in structure, but is substituted by more robust constraint optimization techniques, can detect close tone vibration signatures and takes less computation time than EEMD [13].

In this work, first, we will show by simulated signals the advantages of VMD compared to EEMD in the detection of impulses in the case of variable frequency content. Then, we will use VMD to analyze a one stage bevel gear transmission subjected to time varying loading conditions and in the presence of a local damage.

The structure of the paper is as following: Section 2 introduces the basics of VMD. In Section 3, we present simulated signals to illustrate the non stationary phenomena due to the variation of the speed; these signals are analyzed by using the two methods EEMD and VMD. In Section 4, the VMD method is applied for gearbox faults diagnosis. In Section 5, a conclusion of this paper is given.

\section{Variational mode decomposition (VMD)}

The purpose of VMD [13] is:

- Decompose a signal $x(t)$ into an ensemble of bandlimited intrinsic mode functions $M_{i}$ that have specific sparsity properties while reproducing the input signal. The sparsity prior of each mode was chosen to be its bandwidth.

- Each mode has limited bandwidth.

- Replace the empirical shifting process of EMD by a classical alternate direction method of multipliers (ADMM).

In order to assess the bandwidth of a mode, the following steps were proposed:

1) For each mode $M_{k}$, compute the associated analytic signal by means of the Hilbert transform in order to obtain a unilateral frequency spectrum.

2) For each mode, shift the mode's frequency spectrum to "baseband", by mixing with an exponential tuned to the respective estimated center frequency $\omega_{k}$.

3) The bandwidth was estimated through the $H^{1}$ Gaussian smoothness of the demodulated signal, i.e. the squared $\mathrm{L}^{2}$-norm of the gradient.

4) The reconstruction constraints used in VMD are based on the combination of the two terms: the quadratic penalty term and the Lagrangian multipliers $\lambda$, where their update parameter is $\tau$.

The variational mode decomposition model is essentially based on the three concepts:

a) On the concepts of the Wiener filter for denoising, where the variance of the white noise is $\alpha$. 

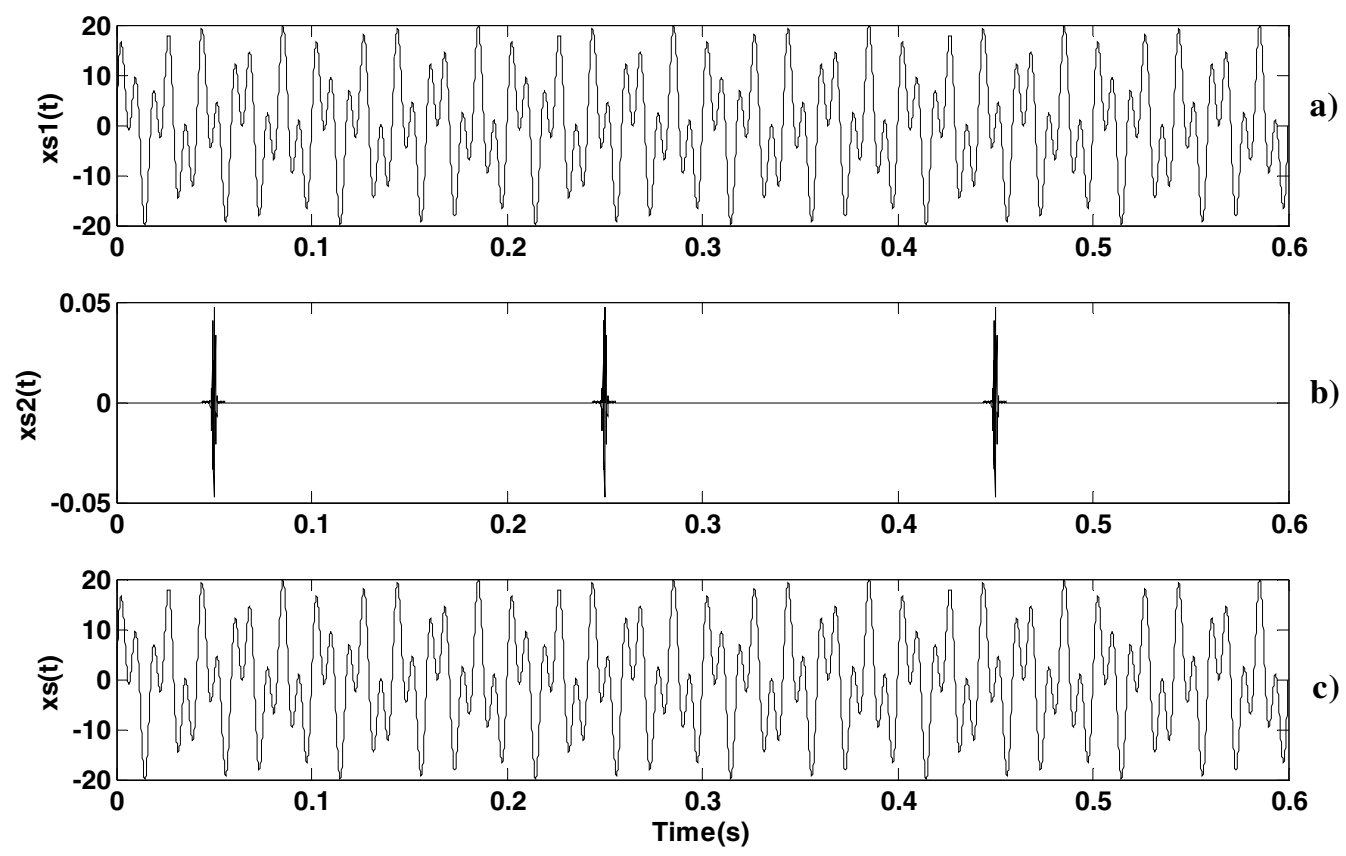

Fig. 2. The stationary simulated signal, (a) the periodic component, (b) the transient component, (c) the signal.

b) The Hilbert transform which is used in the construction of a single-side band analytic signal.

c) On the frequency shifting that is obtained by multiplication with pure complex harmonics.

The VMD algorithm is given in Figure 1. The advantages of the VMD method are:

a) The relative reconstruction error is largely independent of the harmonic's frequency. Moreover, the relative error is nicely controlled by the tolerance level $\epsilon$.

b) The proposed VMD achieves good tones separation $[13]$.

\section{Simulation}

In order to verify the validity of the VMD and to compare the two methods, EEMD and VMD, we suggest analyzing two simulated signals. In the first one, the frequency content is constant, whereas it is variable for the second signal. This comparison will highlight the advantages of applying VMD in fault diagnosis when nonstationary conditions occur.

\subsection{Stationary case}

Let us consider a vibration signal $x_{s}(t)$ consisting of two components $x_{s 1}(t)$ and $x_{s 2}(t)$ (Fig. 2), where $x_{s 1}(t)$ represents the periodic components in the vibration signal, given by:

$$
x_{s 1}(t)=10\left(\sin \left(2 \pi f_{1} t\right)+\sin \left(2 \pi f_{2} t\right)\right) \quad 0 \leq t \leq 0.6 \mathrm{~s} .
$$

$x_{s 2}(t)$ represents the transient component produced by the fault, given by:

$$
x_{s 2}(t)=A_{1}(t) A_{2}(t) \quad 0 \leq t \leq 0.6 \mathrm{~s}
$$

where,

$$
A_{1}(t)=0.05 \sin (2 \pi 2000 t)
$$

and

$$
\begin{aligned}
A_{2}(t)= & \exp \left(\frac{(t-0.05)^{2}}{10^{6}}\right)+\exp \left(\frac{(t-0.25)^{2}}{10^{6}}\right) \\
& +\exp \left(\frac{(t-0.45)^{2}}{10^{6}}\right)
\end{aligned}
$$

The sampling period is: $T e=0.0001 \mathrm{~s}$.

The impacts occur at the times of $0.05 \mathrm{~s}, 0.25 \mathrm{~s}$ and $0.45 \mathrm{~s}$.

The FFT spectrum of the signal is given in Figure 3. It shows only the frequencies which represent the periodic part of the signal. We cannot observe any frequency that represents the transient part given by $x_{s 2}(t)$.

By using EEMD, the signal $x_{s}(t)$ is decomposed using the ensemble number of 100 and for different added noise amplitudes $(0,0.001$ and 0.006$)$ time standard deviation of the signal. The results obtained are shown in Figures 4-6. From these figures, it can be noticed that the components included in the signal are perfectly decomposed into separate IMFs only when we use amplitude of noise different to zero. For Figure 4 we haven't detect the impulses because we haven't use any noise in the decomposition which is similar to the idea of EMD. In Figures 5 and 6, the first IMF corresponds to the transient component; the second IMF, the third IMF and the fourth IMF indicate the periodic components, and the last one corresponds to the residue. But when we use a high amplitude 


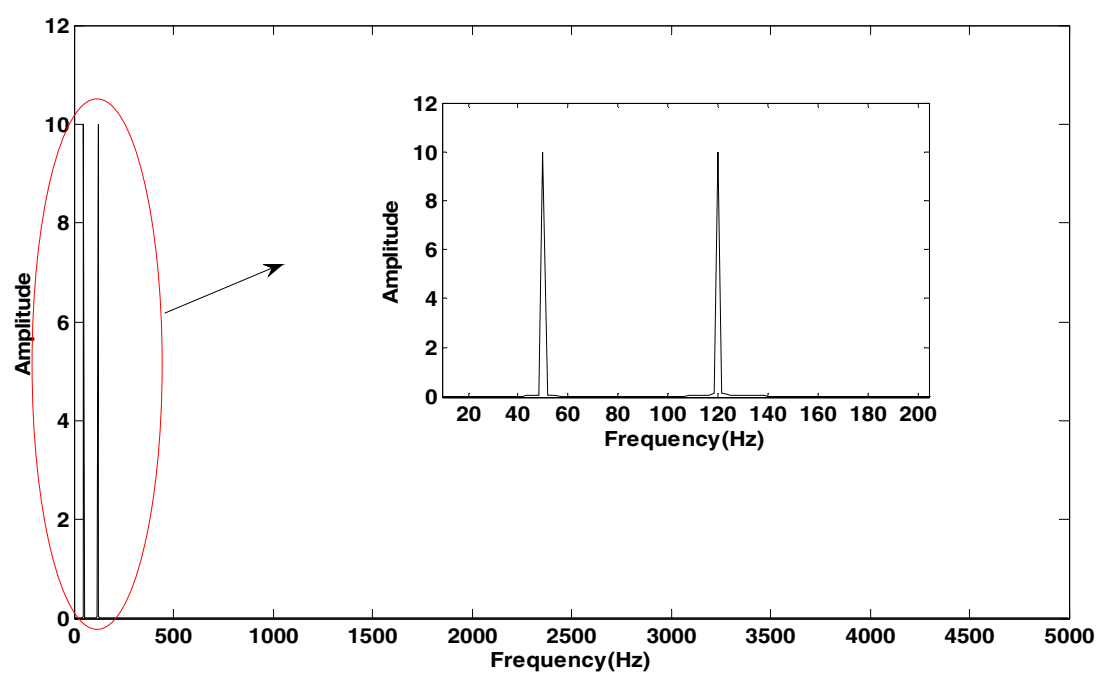

Fig. 3. The spectrum of the stationary signal.
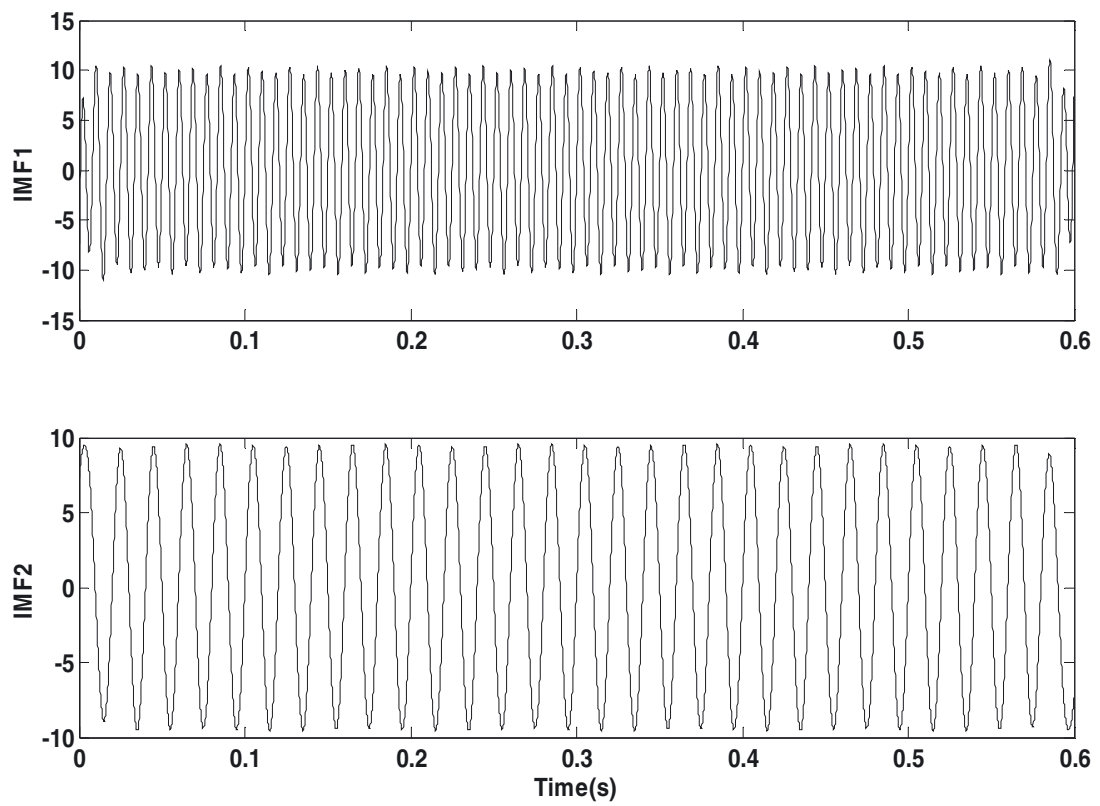

Fig. 4. Results of EEMD for stationary signal (added noise amplitude is zero).

of noise (Fig. 6) the impulses are masked by the noise. Then, the different components embedded in the signal are extracted perfectly if controlled noise is used in the EEMD method.

By using VMD, the signal $x_{s}(t)$ is decomposed using $\alpha=200, K=3, \tau=0$ and $\epsilon=10^{-7}$. The results obtained are shown in Figure 7 in which it can be observed that the components included in the signal are perfectly decomposed into separate VMFs. The first and the second VMFs indicate the periodic components, whereas the third VMF corresponds to the transient component. Then, the different components embedded in the signal are also extracted perfectly using the VMD method.

If we compare the results, we can observe that the first IMF (transient component) given by EEMD is noisy, because the noise used in the EEMD algorithm, (noise amplitude generally greater than 0), occurs generally in the first IMF (Figs. 5 and 6), we can observe that this noise does not exist in the first VMF (transient component) (Fig. 7) because the VMD does not use any noise in its algorithm.

\subsection{Nonstationary case}

Now if we take the stationary signal given in Equation (5) and consider a linear variation of its frequency according to time as:

$f_{1 N s}=f_{1} t$ and $f_{2 N s}=f_{2} t$, the periodic component becomes a chirp signal as following:

$$
\begin{array}{r}
x_{N s 1}(t)=10\left(\sin \left(2 \pi f_{1} t^{2}\right)+\sin \left(2 \pi f_{2} t^{2}\right)\right) \\
0 \leq t \leq 0.6 \mathrm{~s}
\end{array}
$$



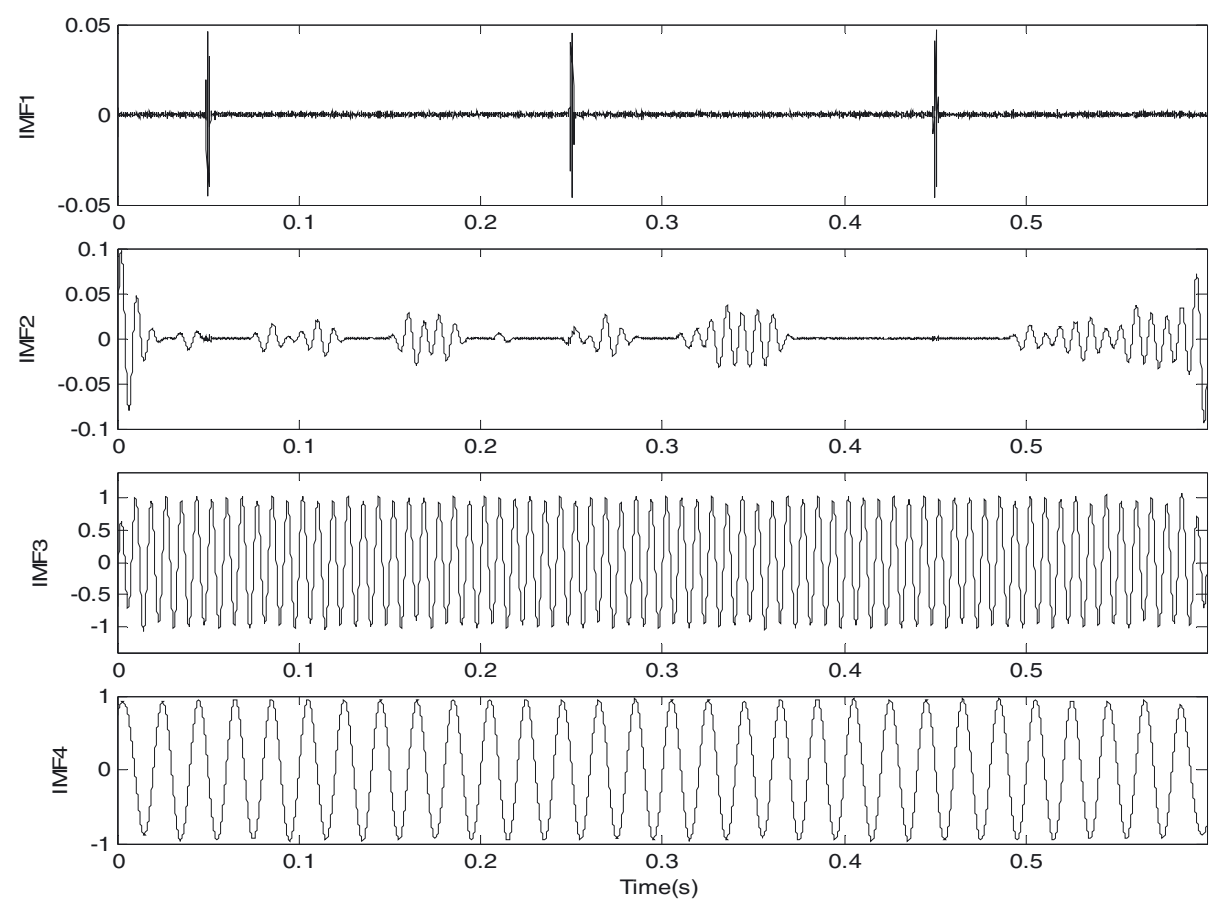

Fig. 5. Results of EEMD for stationary signal (added noise amplitude is 0.001).
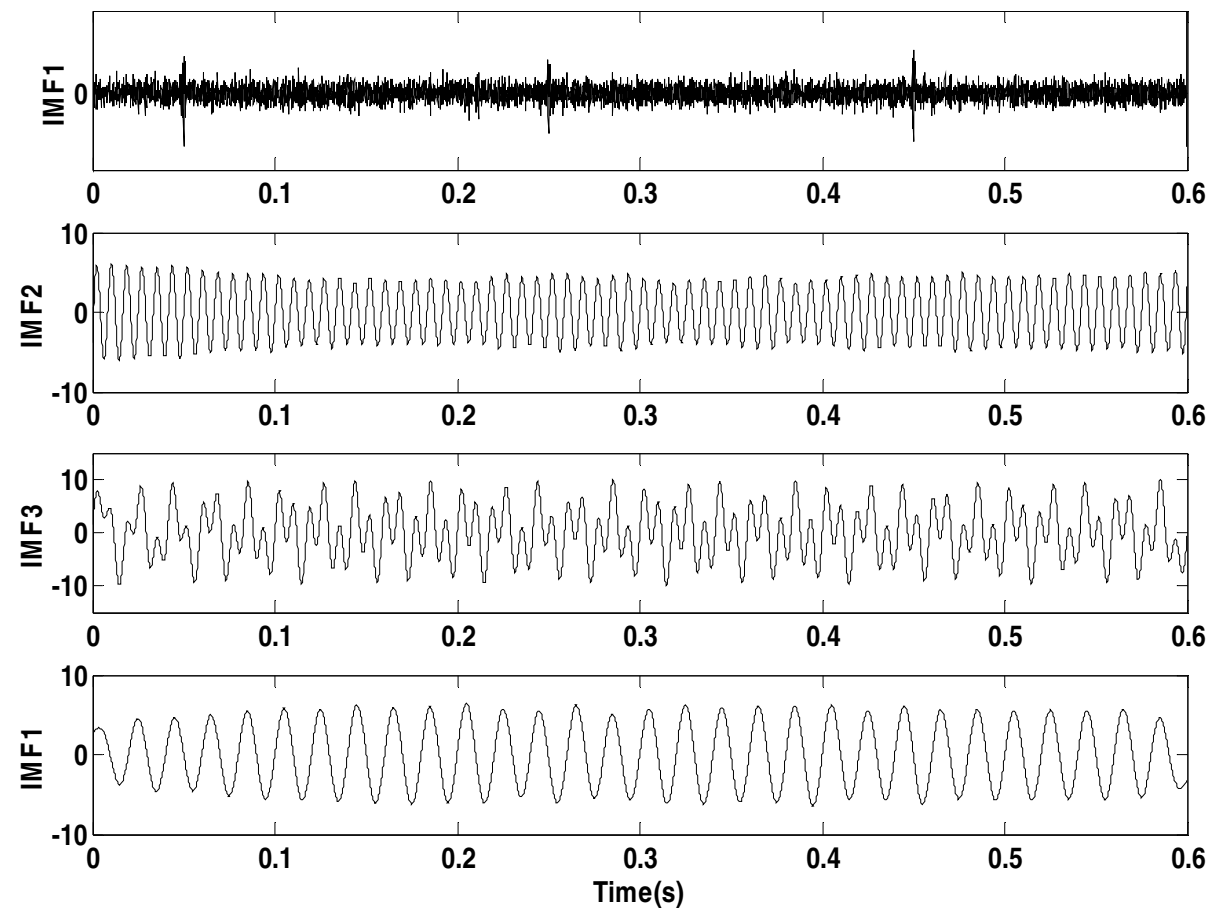

Fig. 6. Results of EEMD for stationary signal (added noise amplitude is 0.006).

Then, the resulting nonstationary signal $x_{N s}(t)$ shown in Figure 8 will be composed of the two components $x_{N s 1}(t)$ and $x_{N s 2}(t)$ (transient component). By using EEMD, the signal $x_{N s}(t)$ is decomposed into eleven IMFs. Figure 9 shows only the first five IMFs which give the important information about the signal. The lasts IMFs represent only the trend of the signal.
The results shown in Figure 9 give an idea on the problem of the mode mixing and the end effect (the region surrounded by black) presented in the first IMF, this problem was not completely removed by the use of the EEMD. Figure 10 shows a zoom on the first IMF and gives an idea on the presence of noise which is generally arisen in the first IMF. The impulses (the region surrounded by the red) are generally obtained also in the first IMF. These 

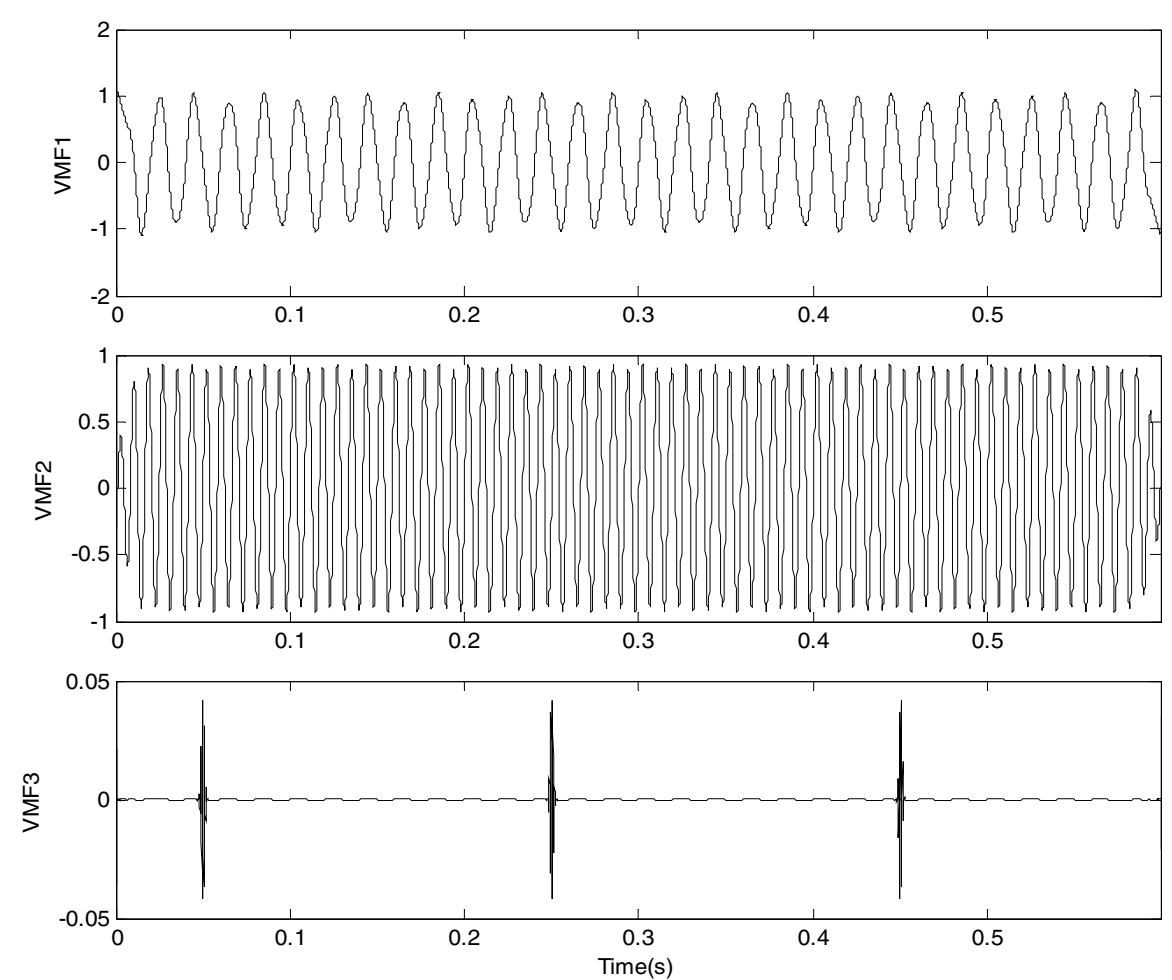

Fig. 7. Results of VMD for stationary signal.
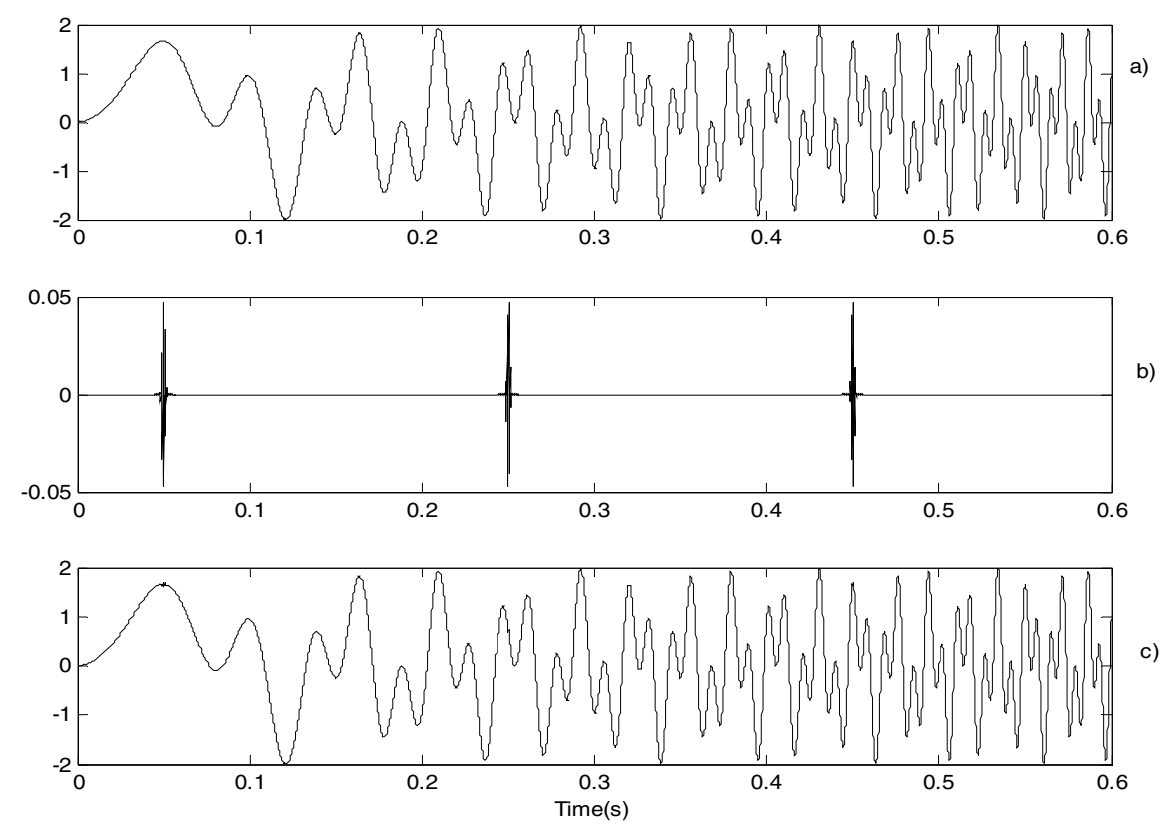

Fig. 8. The nonstationary simulated signal, (a) the chirp, (b) the transient component, (c) the signal.

impulses are indicator of the presence of fault. If the impulses amplitude is too small, the presence of noise and other parts of the signal due to mode mixing in the first IMF can mask the impulses.

When we apply VMD method to analyze the signal, we obtain six VMFs that correspond to several frequency bands as shown in Figure 11. As can be clearly seen, VMD achieves good modes separation without mode mixing phenomenon and noise in the first mode which allows a good detection of the impulses.

\section{Application}

In order to highlight the efficiency of VMD method, we implement it on simulations issued from a dynamic model of a gear transmission running under non-stationary 


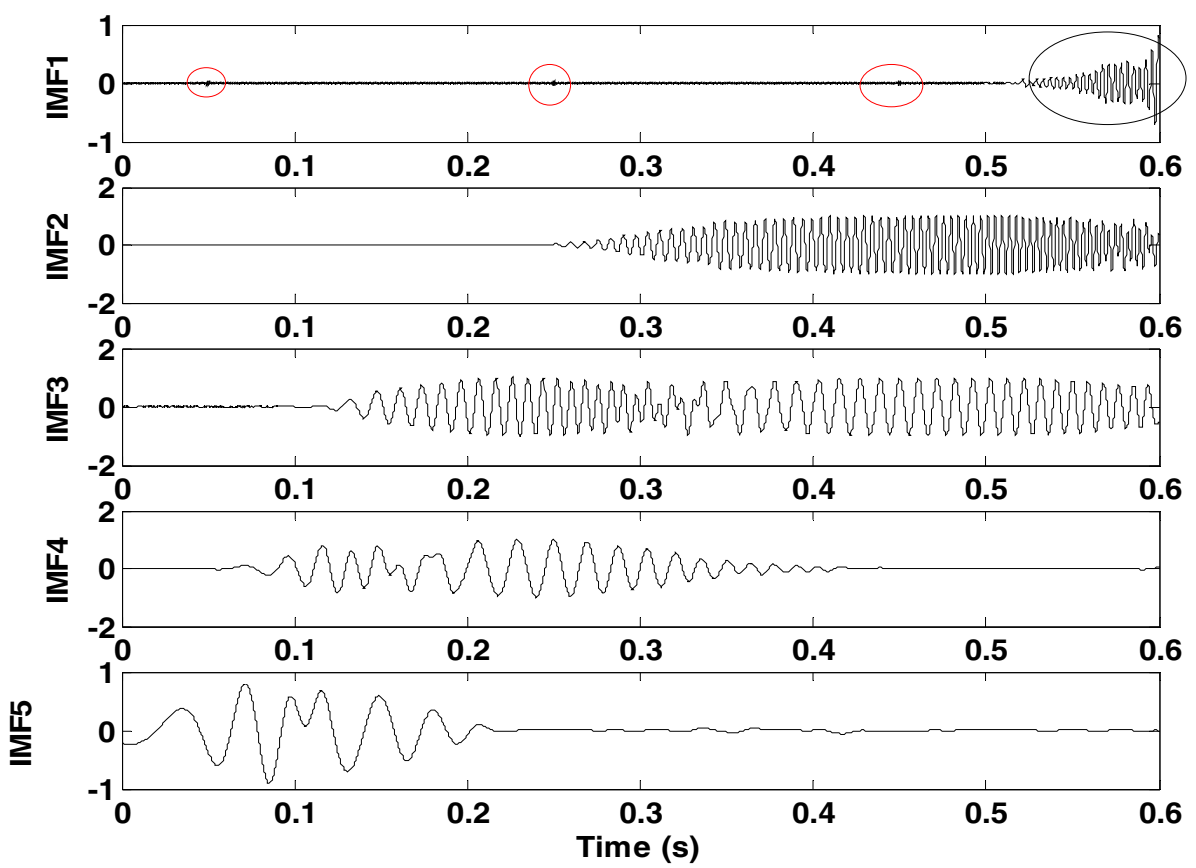

Fig. 9. Results of EEMD for nonstationary signal.

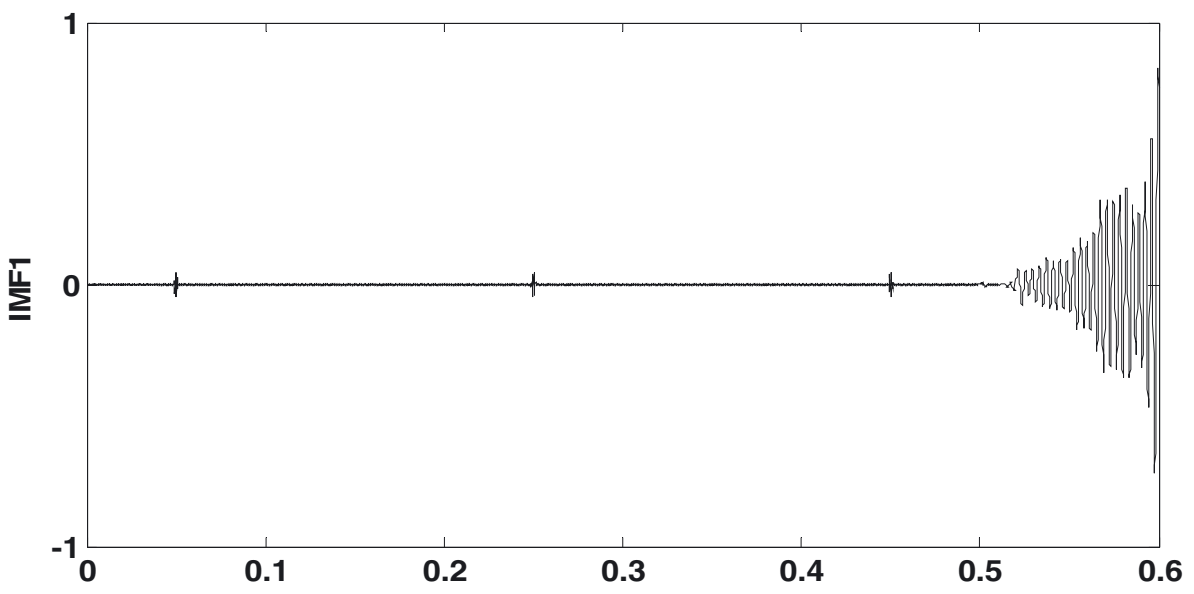

Fig. 10. The first IMF zoomed.

conditions (variable load and speed). It was shown in literature that the analysis of simulated vibration signals from gear models using Wigner Ville [20] or spectrogram $[21,22]$ does not make it possible to detect teeth defects at an early stage. This is mainly caused by the fact that impacts induced by this localized defect are masked by the part of the signal with simultaneous amplitude and frequency modulation induced by speed and load variation. A bevel gear transmission model is considered. It is driven by a squirrel cage electric motor [20] and have the characteristics given in Table 1 . The transmission is loaded with a torque having sawtooth shape with frequency $f_{\mathrm{L}}=5 \mathrm{~Hz}$ as presented in Figure 12a.

The variation of load leads to a fluctuation in the rotational speed (Fig. 12b) and a variation of the gearmesh frequency. The mean value of the motor rotational speed is $n_{\mathrm{r}}=1130 \mathrm{rpm}$ which corresponds to a mean gearmesh frequency $f_{\mathrm{gm}}=263 \mathrm{~Hz}$. The sampling frequency is $30800 \mathrm{~Hz}$ for all signals. A crack in one pinion tooth is simulated by a periodic decrease in the gearmesh stiffness function corresponding to the mesh of the defected tooth. The acceleration signals for healthy gear and faulty gear for early and advanced stage are given in Figure 13. From literature the spectrum of a gear transmission submitted to a constant load is dominated only by the gearmesh frequency and its harmonics. However in the case with time varying load, a family of sidebands appeared around the gearmesh frequency $f_{\mathrm{gm}}$ and its harmonics induced by the non uniformity of the gearmesh period (Fig. 14) and this can be thought to be a frequency modulation of the gearmesh stiffness.

The zoom of this spectrum around the mesh frequency shows many side lines around of this frequency, which indicate a frequency modulation. 


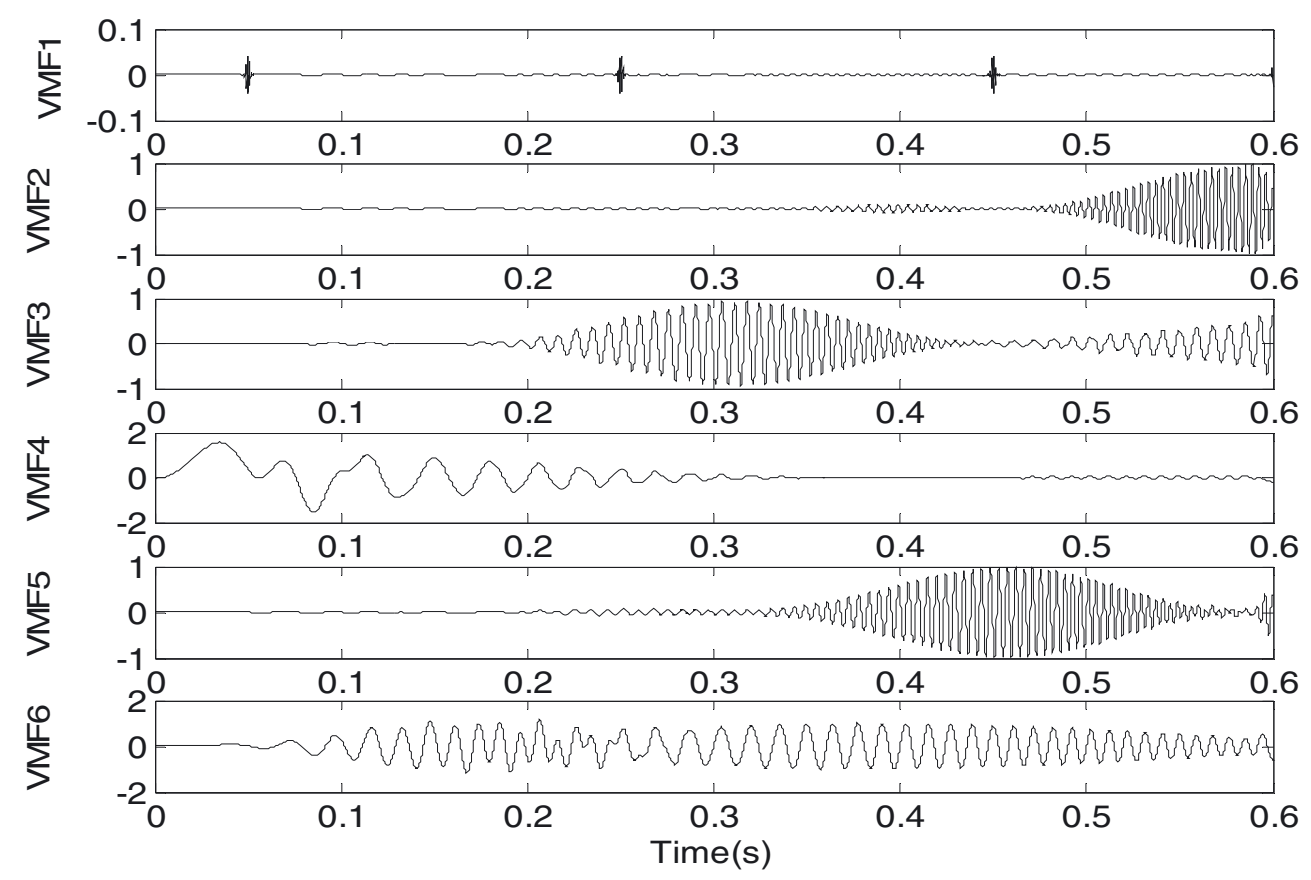

Fig. 11. Results of VMD for non-stationary signal.

Table 1. Parameters of the bevel gear transmission [23].

\begin{tabular}{lcc}
\hline & Pinion & Gear \\
\hline Pitch $(\mathrm{m})$ & \multicolumn{2}{c}{0.0095} \\
Number of teeth $Z$ & 14 & 45 \\
Teeth width $b(\mathrm{~m})$ & \multicolumn{2}{c}{0.064} \\
Pressure angle $\alpha$ & \multicolumn{2}{c}{20} \\
Mass $(\mathrm{kg})$ & 24.7 & 122.6 \\
Moment of inertia $\left(\mathrm{kg} . \mathrm{m}^{2}\right)$ & 0.0585 & 1.91 \\
Axial stiffness $k_{x 1}, k_{y 2}\left(\mathrm{~N} . \mathrm{m}^{-1}\right)$ & $10^{9}$ & $2.3 \times 10^{9}$ \\
Lateral stiffness $k_{y 1}, k_{z 1}, k_{x 2}, k_{z 2}\left(\mathrm{~N} . \mathrm{m}^{-1}\right)$ & $8.8 \times 10^{9}$ & $1.3 \times 10^{10}$ \\
Torsional stiffness $k_{\theta 1}, k_{\theta 2}\left(\mathrm{Nm} \cdot \mathrm{d}^{-1}\right)$ & $1.2 \times 10^{4}$ & $7.4 \times 10^{4}$ \\
\hline
\end{tabular}

It is well-known from references [3-5] that the frequency content of a faulty gear in stationary conditions is dominated mainly by the mesh frequency and its harmonics surrounded by sidebands. From the presented zoomed spectrum (Fig. 14a) for healthy case, it is well observed the high activity of sidebands around mesh frequency and its harmonics. Sidebands can be a serious indicator of the presence of some kind of local damage on teeth or in bearings in stationary conditions. So it can be concluded that for non-stationary operating conditions separated time and frequency analysis for the studied case may induce in error during condition monitoring process.

In order to overcome this difficulty, we propose to use VMD to analyze such signals. Figures $15-17$ present the VMFs of each studied signal. The first VMF of the signal without defect show only the position of the variation of the load, the spectrogram of this VMF gives an idea on the variation of the load (Fig. 18) but the first VMFs of the signals of the faulty gear show periodical impulses. The spectrograms (Figs. 19 and 20) of these VMFs give information of the position of the maximum load and show clearly the position of the impulses due to the fault. The periodicity of the defect can be clearly observed: it is that of the faulty pinion $(T=0.05 \mathrm{~Hz})$.

A statistical study based first, on calculation of the correlation between the signals, (Healthy gear, faulty gear for early and advanced stage), and modes (VMFs) and secondly, on the kurtosis of each VMFs, shows (Tab. 2) that correlation's coefficients do not give any significant information about the faulty gear, however the last three VMFs give more information than the first ones whereas their correlation's coefficients are less than 0.1 .

But for kurtosis we can see that the kurtosis of each signal is around 3, this value doesn't indicate any fault, however the kurtosis of the three last VMFs, which correspond to the two types of fault is very large compared to the ones which correspond to the healthy gears. 
H. Mahgoun et al.: Mechanics \& Industry 17, 207 (2016)
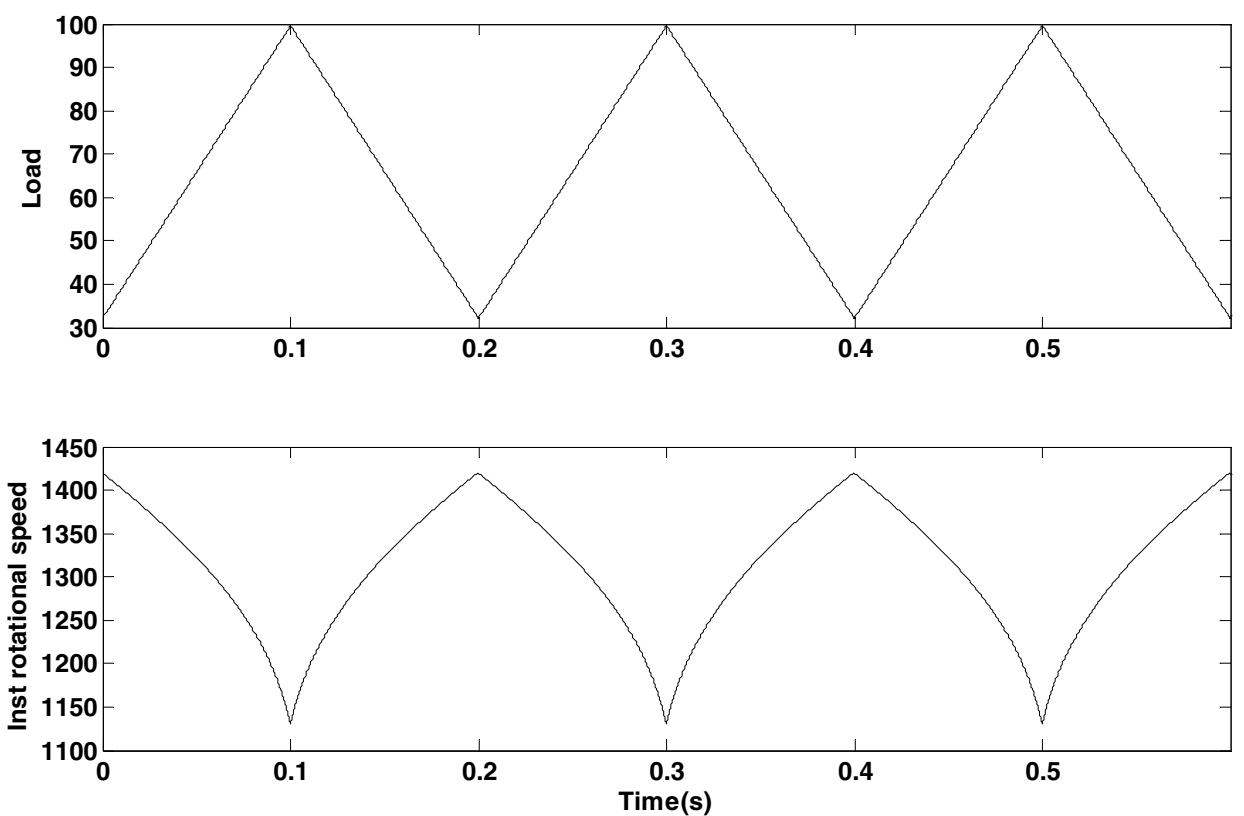

Fig. 12. (a) Evolution of the applied load, (b) evolution of the instantanous rotational speed.
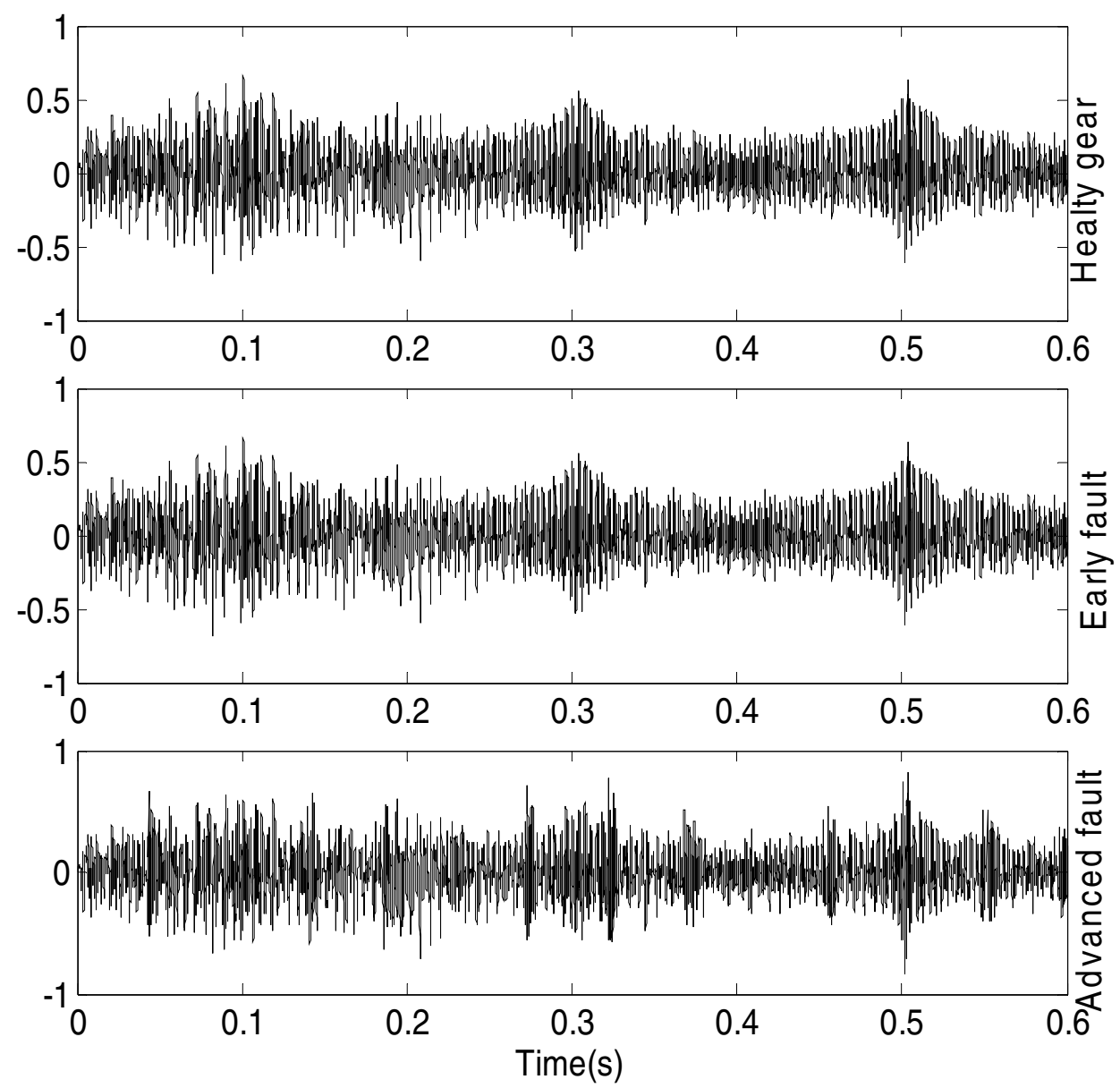

Fig. 13. Acceleration signals (a) healthy gear, (b) and (c) faulty gear. 
H. Mahgoun et al.: Mechanics \& Industry 17, 207 (2016)
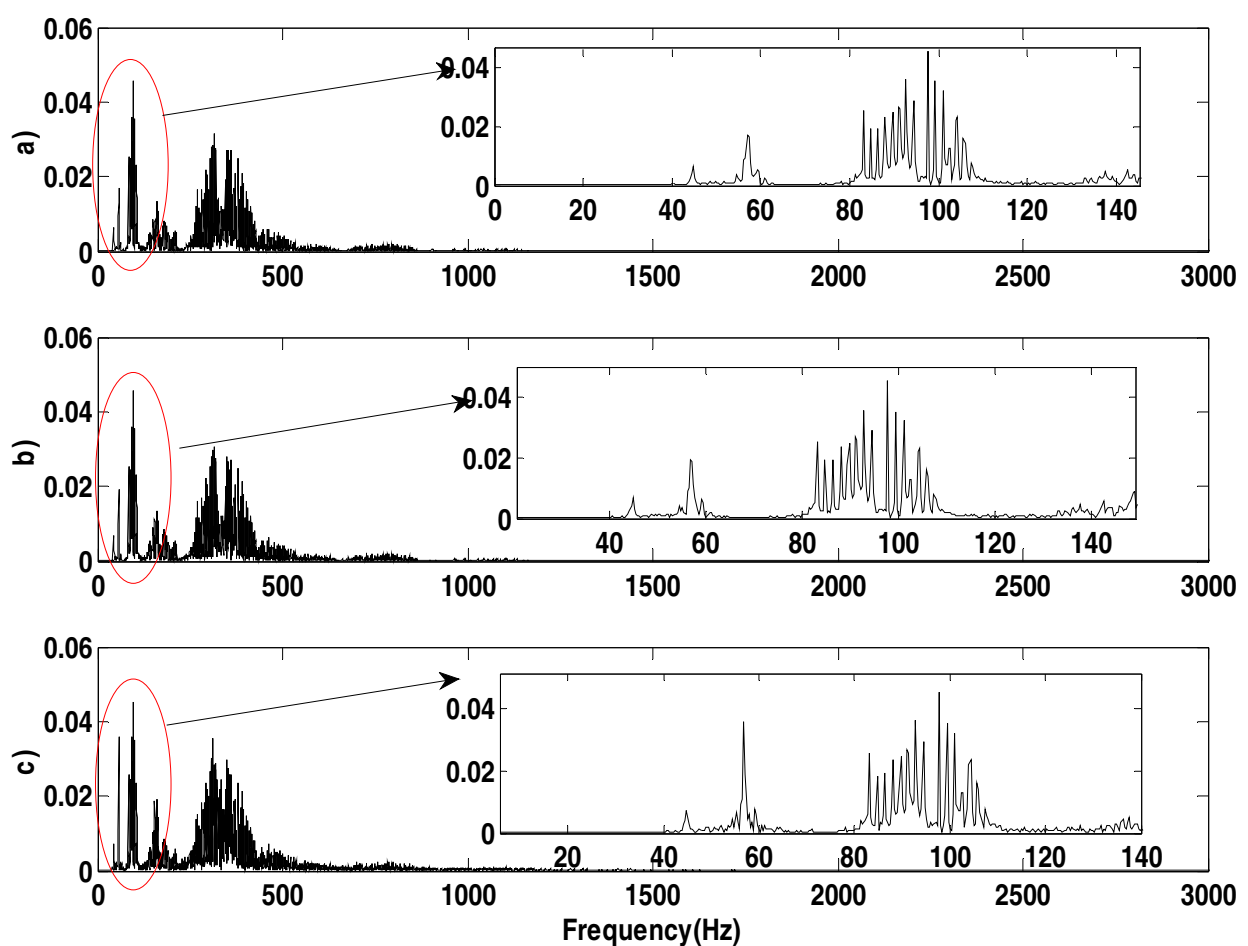

Fig. 14. Spectrum of the acceleration signals (a) healthy gear, (b) and (c) faulty gear.
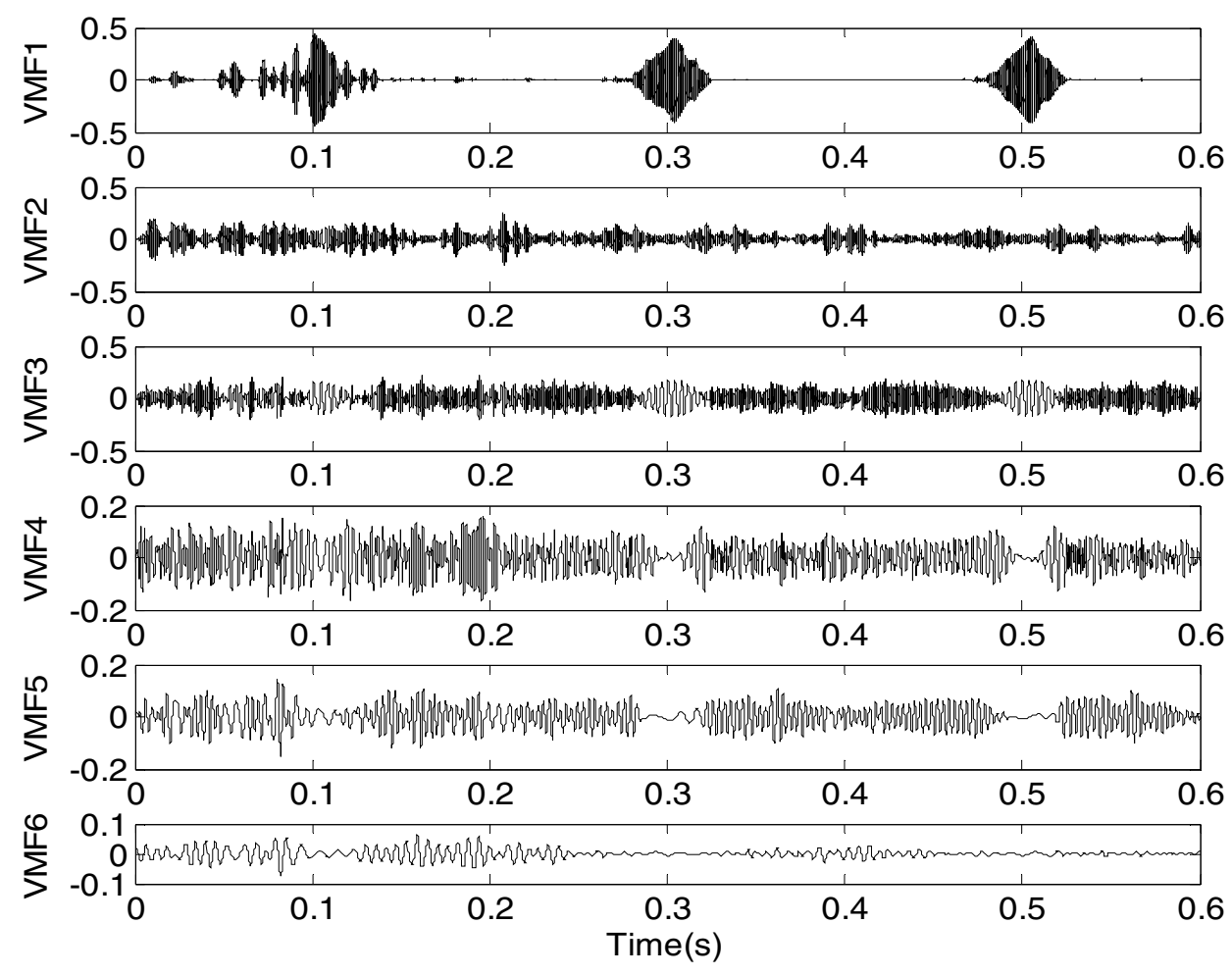

Fig. 15. VMD of the acceleration signal for healthy gear. 
H. Mahgoun et al.: Mechanics \& Industry 17, 207 (2016)
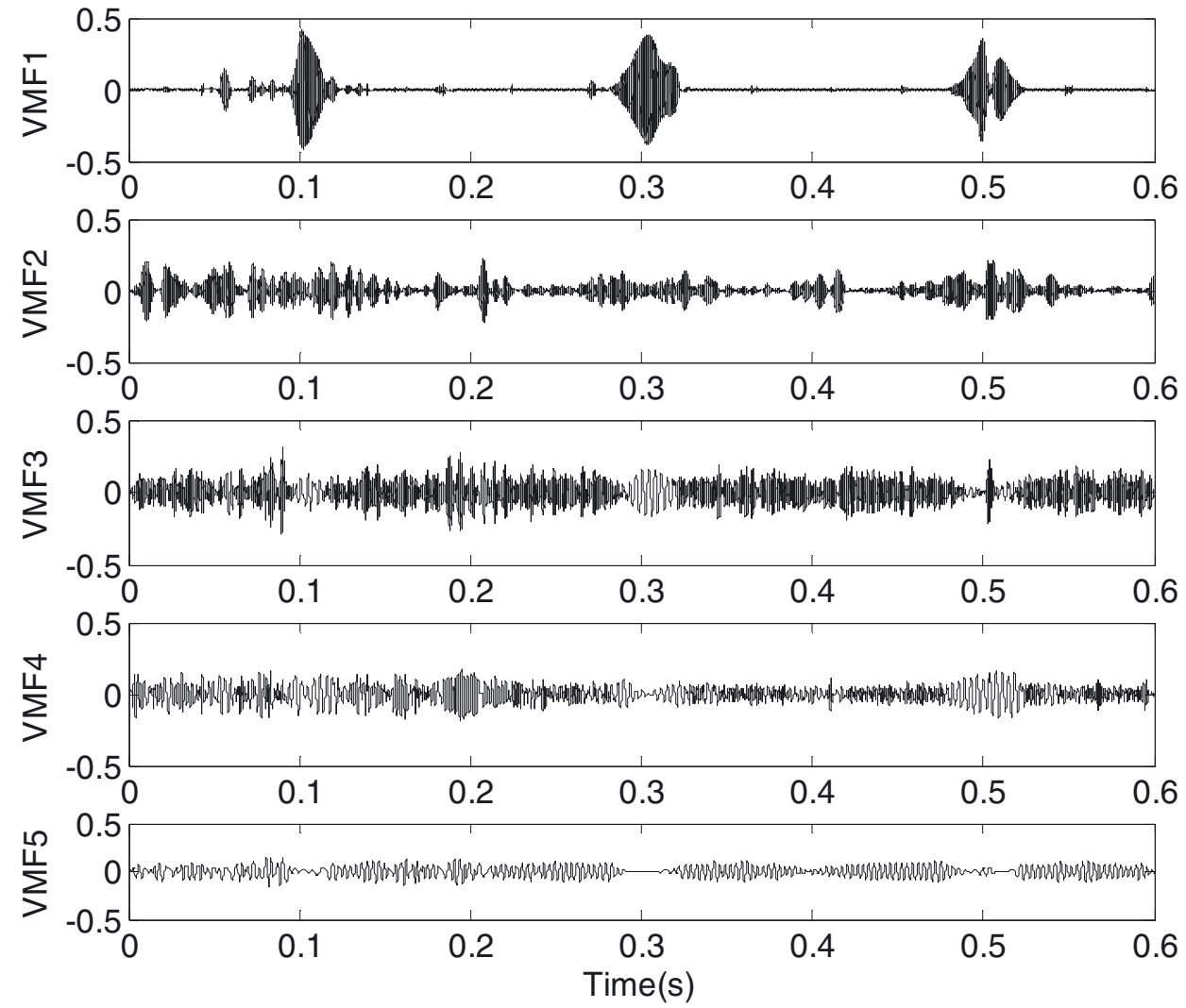

Fig. 16. VMD of the acceleration signal for early fault.
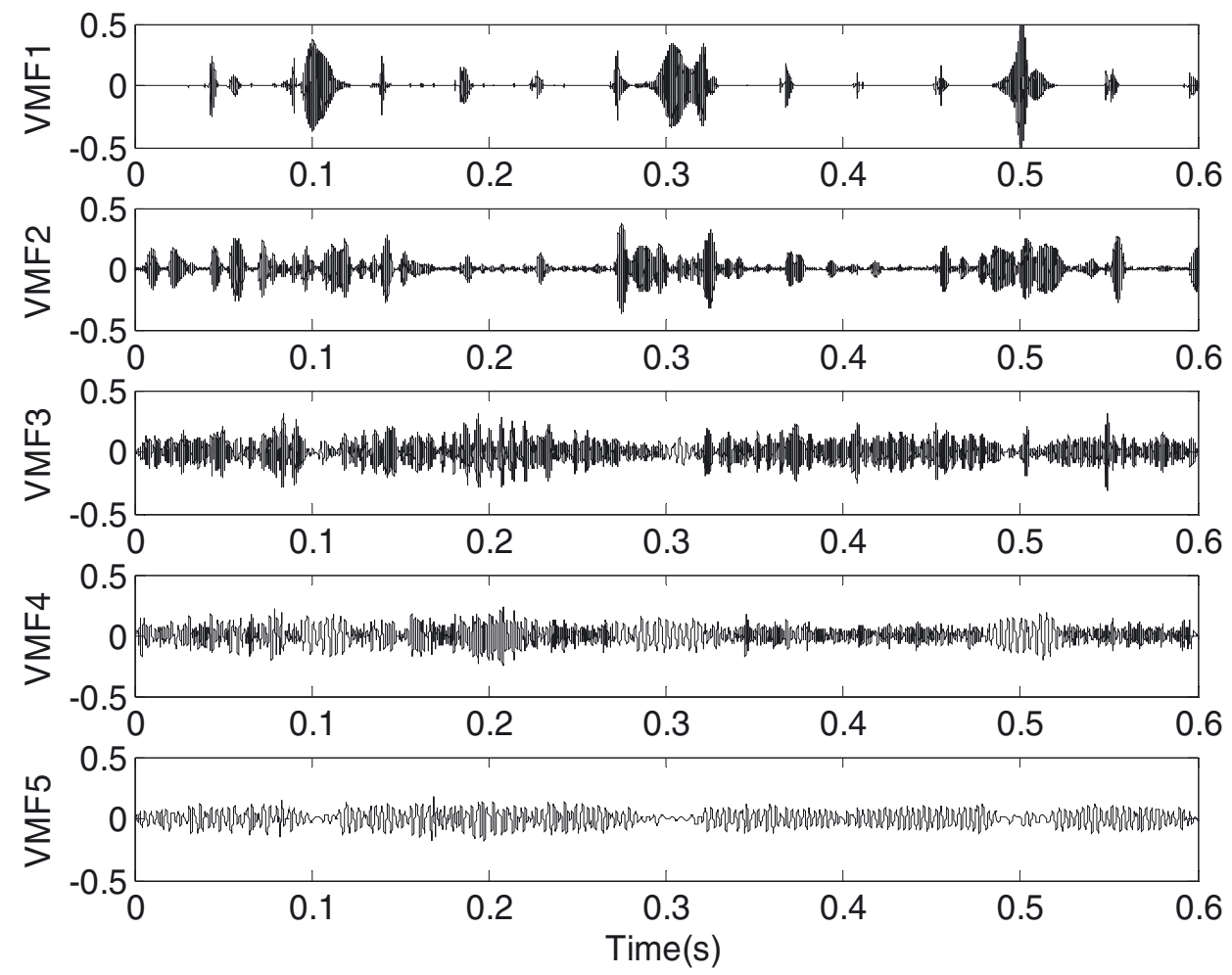

Fig. 17. VMD of the acceleration signal for advanced fault. 
H. Mahgoun et al.: Mechanics \& Industry 17, 207 (2016)
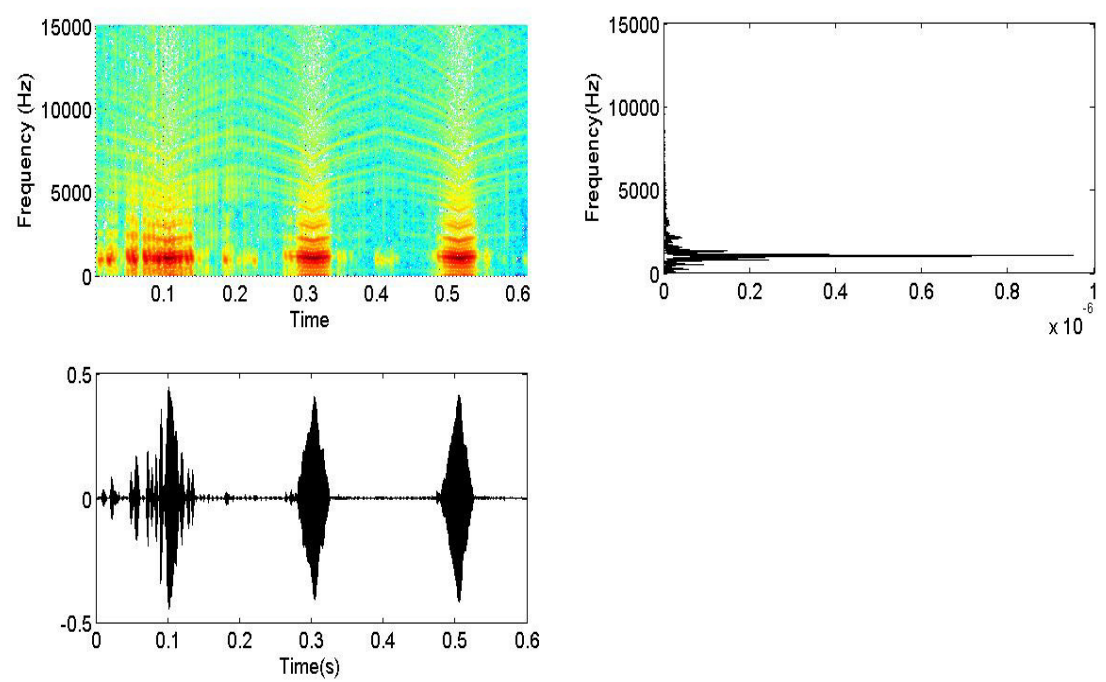

Fig. 18. Spectrogram and spectrum of the first VMF for healthy gear.
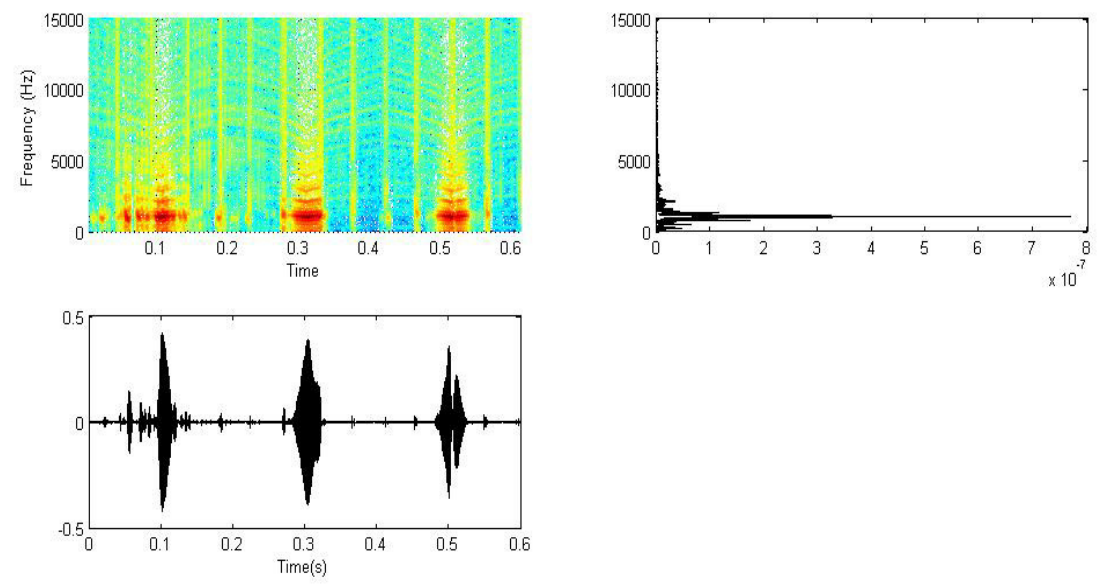

Fig. 19. Spectrogram and spectrum of the first VMF for early fault.
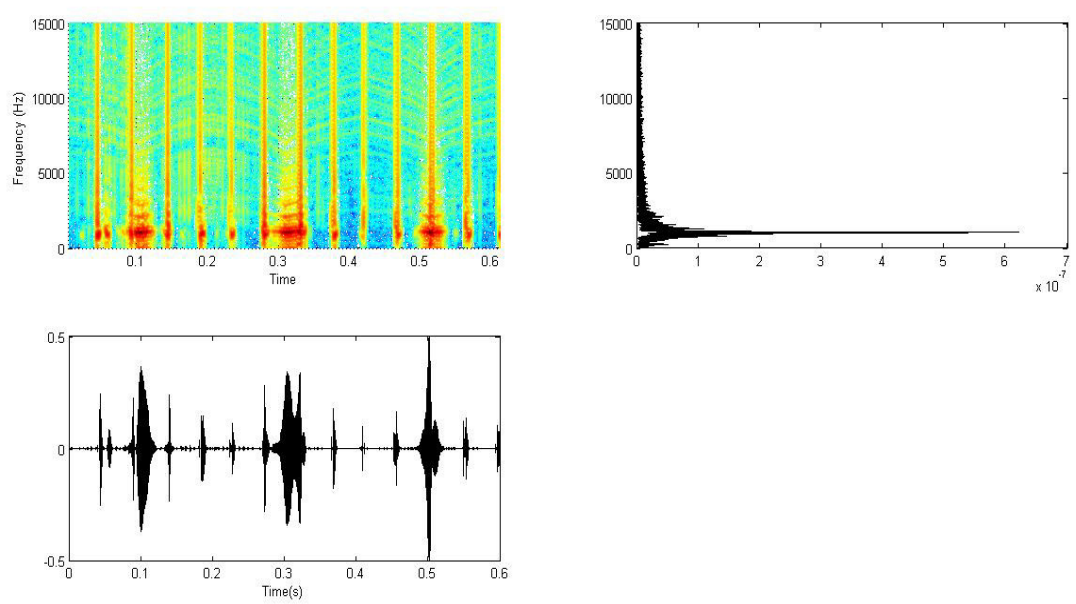

Fig. 20. Spectrogram and spectrum of the first VMF for advanced fault. 
Table 2. Statistical study.

\begin{tabular}{|c|c|c|c|c|c|c|}
\hline \multirow{3}{*}{$\begin{array}{l}\text { Signal } \\
\text { VMFs }\end{array}$} & \multicolumn{2}{|c|}{ healthy gear } & \multicolumn{2}{|c|}{ Faulty gear (early) } & \multicolumn{2}{|c|}{ Faulty gear (advanced) } \\
\hline & \multirow{2}{*}{ Correlation coeff. } & Kurtosis & \multirow{2}{*}{ Correlation coeff. } & Kurtosis & \multirow{2}{*}{ Correlation coeff. } & \multirow{2}{*}{$\begin{array}{c}\text { Kurtosis } \\
3.26\end{array}$} \\
\hline & & 3.18 & & 3.14 & & \\
\hline VMF1 & 0.0357 & 5.8504 & 0.0265 & 40.2272 & 0.0330 & 262.1139 \\
\hline VMF2 & 0.0677 & 3.4868 & 0.0466 & 8.4364 & 0.0579 & 90.8767 \\
\hline VMF3 & 0.1183 & 3.3088 & 0.0906 & 4.2081 & 0.1313 & 14.7359 \\
\hline VMF4 & 0.5232 & 2.3124 & 0.1501 & 3.8247 & 0.4893 & 2.4117 \\
\hline VMF5 & 0.3801 & 4.2710 & 0.5527 & 2.4871 & 0.4961 & 2.6189 \\
\hline VMF6 & 0.7232 & 3.1702 & 0.7292 & 2.8372 & 0.7650 & 3.8673 \\
\hline VMF7 & 0.5483 & 2.2762 & 0.6058 & 2.5786 & 0.4609 & 4.3059 \\
\hline
\end{tabular}

\section{Conclusion}

In this study we have used the VMD method to analyze non-stationary signals that give information about the variable conditions such as variable speed and load. The VMD method achieves good tones separation and does not require the use any noise in its implementation. This was one of the drawbacks of EEMD method which needs adding noise leading to probable mask of the impulsions due to impacts that are generated by the presence of a defect.

To detect the fault masked by simultaneous variation of load and presence of defect, VMD showed successful separation of the different modes that correspond to the variation of load and the effect of fault. We have also used the spectrogram to detect the period of the impulses due to the fault, and we have observed that the kurtosis calculated for raw signals cannot give any information about the presence of the defect. However, when we computed the kurtosis after decomposition with VMD which separates the different parts of the signal, we have observed that it can be used as an indicator of the fault in such conditions.

\section{References}

[1] P.D. McFadden, Detecting fatigue cracks in gears by amplitude and phase demodulation of the meshing vibration, Transaction of the ASME, J. Vibr., Acoust. Stress Reab. Design 108 (1986) 165-170

[2] Jiesi Luo, Dejie Yu, Ming Liang, Application of multiscale chirplet path pursuit and fractional Fourier transform for gear fault detection in speed up and speed-down processes, J. Sound Vibr. 331 (2012) 4971-4986

[3] C. Capdessus, M. Sidahmed, Analyse des vibrations d'un engrenage: cepstre, corrélation, spectre, Traitement du signal 8 (1992) 365-371

[4] E.B. Halim, M.A.A. Choudhury, S.L. Shah, M.J. Zuo, Time domain averaging across all scales: A novel method for detection of gearbox faults, Mech. Syst. Signal Process. 22 (2008) 261-278
[5] H. Mahgoun, R.E. Bekka, A. Felkaoui, Gearbox fault diagnosis using ensemble empirical mode decomposition (EEMD) and residual signal, Mechanics \& Industry 13 (2012) 33-44

[6] C. Capdessus, E. Sekko, J. Antoni, Speed transform, a new time-varying frequency analysis technique, CMMNO2013, Ferara (2013)

[7] H. Mahgoun, A. Felkaoui, S. Fedala, F. Chaari, Detection of Gear Faults in Variable Rotating Speed Using EEMD Decomposition and Instantaneous Frequency, 4th International Conference on Condition Monitoring of Machinery in Non-stationary Operations, CMMNO-2014, 15-16 Decmber, 2014 Lyon, France

[8] T.Y. Wu, J.C. Chen, C.C. Wang, Characterization of gear faults invariable rotating speed using Hilbert-Huang Transform and instantaneous dimensionless frequency normalization, Mech. Syst. Signal Process. 30 (2012) 103122

[9] Z. Wu, N.E. Huang, Ensemble empirical mode decomposition: a noise-assisted data analysis method, Adv. Adaptive Data Anal. 1 (2009) 1-41

[10] B. Liu, S. Riemenschneider, Y. Xub, Gearbox fault diagnosis using empirical mode decomposition and Hilbert spectrum, Mech. Syst. Signal Process. 17 (2005) 1-17

[11] T.Y. Wu, Y.L. Chung, Misalignment Diagnosis of Rotating Machinery through Vibration Analysis via Hybrid EEMD and EMD approach, Smart Mater. Struct. 18 (2009)

[12] N.E. Huang, Z. Shen, S.R. Long, The empirical mode decomposition and the Hilbert spectrum for nonlinear and non-stationary time series analysis, in: Proc. Royal Soc. London Ser. 454 (1998) 903-995

[13] K. Dragomiretskiy, D. Zosso, Variational Mode Decomposition, IEEE Trans. Signal Process. 62 (2014) $531-544$

[14] N.E. Huang, M.L. Wu, S.R. Long, A confidence limit for the empirical mode decomposition and Hilbert spectral analysis, Proc. Royal Soc. London 459 (2003) 2317-2345

[15] M.A. Colominas, G. Schlotthauer, M.E. Torres, Improved complete ensemble EMD: A suitable tool for biomedical signal processing, Biomed. Signal Process. Control 14 (2014) 19-29

[16] G. Rilling, P. Flandrin, One or two frequencies? The empirical mode decomposition answers, IEEE Trans. Signal Process. 56 (2008) 85-95 
[17] I. Daubechies, J. Lu, H.-T. Wu, Synchrosqueezed wavelet transforms: An empirical mode decomposition-like tool, J. Appl. Computat. Harmonic Anal. 30 (2011) 243-261

[18] H. Mahgoun, A. Felkaoui, R.E. Bekka, The effect of resampling on the analyses results of ensemble empirical mode decomposition (EEMD), International Conference surveillance 6, Compiègne France, 25-26 October, 2011

[19] G. Rilling, P. Flandrin, Sampling effects on the empirical mode decomposition, Adv. Adapt. Data Anal. 01 (2009) $43-59$

[20] F. Chaari, W. Bartelmus, R. Zimroz, T. Fakhfakh, M. Haddar, Gearbox vibration signal amplitude and frequency modulation, Shock Vibration 19 (2012) 635652

[21] F. Chaari, M.S. Abbes, F.V. Rueda, A.F. del Rincon, M. Haddar, Analysis of planetary gear transmission in nonstationary operations, Front. Mech. Eng. 8 (2013) 88-94

[22] W. Bartelmus, R. Zimroz, Vibration condition monitoring of planetary gearbox under varying external load, Mech. Syst. Signal Process. 23 (2009) 246-257

[23] T. Peng, Coupled multi-body dynamic and vibration analysis of hypoid and bevel geared rotor system, Ph.D. Thesis, University of Cincinnati, United States, 2010 